\title{
Análisis de Contaminantes en la Cuenca del Río Aconcagua en Chile. Evaluación de Riesgo Humano y Ambiental
}

\author{
Margarita Préndez ${ }^{(1)}$ y Víctor Calderón ${ }^{(2)}$ \\ (1) Universidad de Chile, Facultad de Ciencias Químicas y Farmacéuticas, Laboratorio de Química de la \\ Atmósfera, Sergio Livingston 1007, Independencia, Santiago-Chile \\ (2) Universidad de Chile, Magister en Gestión y Planificación Ambiental, Santiago-Chile
}

Recibido Abr. 12, 2012; Aceptado Jun. 07, 2012; Versión final recibida Ago. 01, 2012

\begin{abstract}
Resumen
Se presenta un estudio y análisis de contaminantes del aire en una zona de sostenido desarrollo industrial y agrícola en Chile. El estudio comprende la cuenca del río Aconcagua incluyendo varias comunas de las provincias de Valparaíso, Quillota, San Felipe y Marga Marga. La información obtenida de las redes de monitoreo de calidad de aire reguladas por la autoridad ambiental y sanitaria, durante el período 1998 a 2008, se utilizó para elaborar un método de evaluación cualitativa del riesgo de la contaminación para la salud humana y la vegetación. El método evidenció zonas de alto riesgo para la vegetación y la vida humana y otras que debieran ser declaradas zonas saturadas. También mostró la necesidad de establecer normas primarias y secundarias, en particular para algunos elementos traza considerando la importancia de la zona como exportadora de productos agrícolas.
\end{abstract}

Palabras clave: calidad del aire de la cuenca, contaminantes, riesgo humano, riesgo ambiental, zonificación

\section{Analysis of Pollutants in the Watershed of the Aconcagua River in Chile. Evaluation of Human and Environmental Risks}

\begin{abstract}
This paper presents a study and analysis of air pollutants in an area of permanent industrial and agricultural development in Chile. The study considers the watershed of the Aconcagua river including several cities of the provinces of Valparaiso, Quillota, San Felipe and Marga Marga. The information obtained from monitoring networks of air quality that are regulated by the Chilean health and environmental authorities, during the period 1998 to 2008, was used to develop a qualitative method to assess the risk of contamination to human health and vegetation. The method showed areas of high risk to vegetation and human life and other areas that should be declared as saturated zones. It was also demonstrated that it is necessary to establish primary and secondary standards, particularly for some trace elements considering the importance of the area as an exporter of agricultural products.
\end{abstract}




\section{INTRODUCCIÓN}

La cuenca del Aconcagua, específicamente las comunas de: Concón en la provincia de Valparaíso, Quillota, La Cruz, La Calera, Nogales e Hijuelas en la provincia de Quillota, Llay Llay, Catemu y Panquehue en la provincia de San Felipe y Limache en la provincia del Marga Marga, es una zona relevante para el desarrollo económico de la Región de Valparaíso y del país. La presión antropogénica que ha provocado su sostenido crecimiento, ha causado que la calidad ambiental y en especial la calidad del aire sea un tema sensible para el sector productivo agro-industrial presente en la zona, las autoridades y la comunidad. El Censo 2002 mostraba para la Provincia de Quillota 175.917 habitantes y para la provincia de San Felipe 131.911 habitantes. En ellas destaca la actividad frutícola, especialmente la producción de palta, cítricos y vides, siendo los principales mercados EEUU y Holanda para paltas y EEUU y Corea del Sur para las naranjas (ASIVA, 2011). El estudio realizado por CENMA, (2001), concluye que los principales problemas de la calidad del aire de la zona son las altas concentraciones de material particulado respirable y la alta concentración de $\mathrm{O}_{3}$ en la comuna de Hijuelas.

En la última década las fuentes emisoras industriales de la zona (generación energética, producción de cemento, refinación de petróleo y fundición de cobre) han expandido sus proyectos y modificado sus procesos productivos. La escasez de gas natural y el encarecimiento de los combustibles han inducido al mayor uso de petróleo diesel, carbón de petróleo (petcoke) y combustibles alternativos, configurando un escenario desfavorable para la calidad del aire y por ende para la salud de la población y la vegetación. Paralelamente, las evaluaciones ambientales de proyectos han permitido generar una base de datos a partir de redes de monitoreo de la calidad del aire que abarca, en algunos casos, a una década de mediciones. La Autoridad Sanitaria de cada región (ASR) administra y monitorea la calidad del aire registrada en las estaciones de monitoreo públicas y fiscaliza las redes y/o estaciones de monitoreo privadas. La vigilancia de la calidad secundaria del aire le compete al Servicio Agrícola y Ganadero (SAG).

En líneas generales, las estaciones de monitoreo se asocian a planes de descontaminación o a la ejecución de proyectos de inversión evaluados en el Sistema de Evaluación de Impacto Ambiental (SEIA). El Sistema Nacional de Calidad del Aire (SINCA), permite gestionar la información sobre calidad del aire, coordinado por el Ministerio del Medio Ambiente (ex CONAMA), en conjunto con los organismos competentes en la materia (ASR y SAG). Las emisiones atmosféricas, se encuentran reguladas por las normas de emisión. Dentro de los alcances del Registro de Emisión y Transferencia de Contaminantes (RETC), coordinado por el Ministerio del Medio Ambiente y fiscalizado por la ASR, las empresas deben informar cada año a la autoridad sus emisiones a través de la Declaración de Emisiones (DS 138/2005). Toda esta valiosa información no se encuentra visibilizada a nivel de cuenca y queda fragmentada en las instancias respectivas. El propósito de este trabajo es desarrollar, desde un enfoque de riesgo, un método que dé cuenta del estado de la calidad del aire de la cuenca del Aconcagua y los potenciales efectos sobre los habitantes y la vegetación, incluídos los cultivos de exportación, principalmente paltos, cítricos y vides.

La atmósfera está constituida básicamente por $78 \%$ nitrógeno molecular y casi $21 \%$ oxígeno molecular, además de $0,9 \%$ de argón y niveles traza de una multitud de otras especies químicas que definen las propiedades químicas y radiativas de la atmósfera, e interconectan los sistemas bióticos y abióticos estableciendo las condiciones para la vida (Gallardo, 2006). La alteración de la concentración de dichas trazas por las actividades humanas ha generado procesos de contaminación a diversas escalas espaciales y temporales. A nivel de las ciudades, la contaminación atmosférica se ha transformado en un problema de salud pública (Gramsch et al, 2006; Tak Sun Yu et al, 2012).

Cuando un contaminante entra a la atmósfera su comportamiento es afectado por factores propios de su naturaleza química, sus características fisicoquímicas, la cantidad y frecuencia de emisión, y por factores externos: presencia de otras sustancias en el medio e interacciones con dichas sustancias, factores geográficos y factores meteorológicos como temperatura, humedad relativa, radiación solar, presión atmosférica, velocidad y dirección del viento, entre otros. Como consecuencia, las especies químicas presentes en el aire pueden ser muy diferentes de las emitidas, así como sus efectos sobre la salud humana y ambiental (Seguel et al, 2009). Además, el alcance de los contaminantes bajo ciertas condiciones de ventilación puede ir desde escalas espaciales locales a regionales e incluso globales.

De las especies traza presentes en la atmósfera sólo algunas han sido de interés regulatorio por sus efectos en receptores humanos y vegetales. Entre estos se cuentan MP10 y MP2,5 (material particulado de diámetro aerodinámico $<10$ y a $2,5 \mu \mathrm{m}$, respectivamente), dióxido de azufre $\left(\mathrm{SO}_{2}\right)$, óxidos de nitrógeno $\left(\mathrm{NO}_{\mathrm{x}}\right)$, monóxido de carbono $(\mathrm{CO})$, hidrocarburos totales $(\mathrm{HCT})$ y ozono $\left(\mathrm{O}_{3}\right)$. Entre los hidrocarburos están el metano (corresponde al $40 \%-80 \%$ de los hidrocarburos presentes en una atmósfera urbana) y los alquenos y acetilenos, los hidrocarburos aromáticos, tales como el benceno, tolueno y xileno y los terpenos, hidrocarburos volátiles emitidos de fuentes naturales (biogénicos), todos ellos agrupados como Hidrocarburos No Metánicos (HCNM) 
(Rana, 2006). Ciertos tipos de HCNM reaccionan en presencia de radiación solar con los $\mathrm{NO}_{\mathrm{x}}$ originando oxidantes fotoquímicos tales como el $\mathrm{O}_{3}$ y la familia de los peroxiacil nitratos (PANs), compuestos de alta toxicidad (Préndez, 1993).

En Chile se han establecido normas primarias de calidad del aire para los denominados contaminantes criterio: $\mathrm{SO}_{2}, \mathrm{NO}_{2}, \mathrm{MP}_{10}, \mathrm{O}_{3}$ y $\mathrm{CO}$, y una norma para el $\mathrm{Pb}$ presente en el MP10. Como normas secundarias, para la protección de especies vegetales y ecosistemas, sólo están normados el $\mathrm{SO}_{2}$ y el material particulado sedimentable (diámetro aerodinámico superior a $45 \mu \mathrm{m}$ ), esto último sólo aplicable en la cuenca del Huasco en la región de Atacama. La Organización Mundial de la Salud (OMS), 2006, sobre la base de diversos estudios epidemiológicos, evidencia los efectos perjudiciales que tiene la exposición a gases como $\mathrm{SO}_{2}, \mathrm{NO}_{\mathrm{x}}$ y $\mathrm{O}_{3}$, así como también al MP10 y a algunos elementos traza presentes en él, tales como As y Pb, entre otros y recomienda valores límite.

Los gases contaminantes primarios $\mathrm{SO}_{2}$ (incoloro, irritante) y $\mathrm{NO}_{2}$ (amarillo-café, irritante) y el secundario $\mathrm{O}_{3}$ (incoloro, oxidante fuerte) generan efectos negativos agudos y crónicos sobre la salud humana y la vegetación. En una atmósfera oxidante los gases primarios conducen a la formación de sus respectivos ácidos y/o sales derivadas, los cuales afectan otros componentes ambientales: suelo, cultivos, aguas superficiales, viviendas, entre otros (Rana, 2006; OMS, 2006). $\mathrm{El}_{3}$ derivado de reacciones básicamente fotoquímicas en que participan los $\mathrm{NO}_{x}$ y compuestos orgánicos, afecta también a la salud humana (Cakmak et al., 2007) y ambiental, siendo la vegetación particularmente sensible, generando síntomas crónicos y agudos que reducen la fotosíntesis (Calfapietra et al., 2009), afectando productividad y calidad (WHO, 2000).

\section{MATERIAL PARTICULADO}

Diversos estudios epidemiológicos, evidencian efectos adversos del material particulado (MP) en la salud de las personas (Fernández et al., 2004; Tak Sun Yu, 2012). Dichos efectos son amplios, particularmente en los sistemas respiratorios y cardiovascular, tanto a exposiciones leves como prolongadas (OMS, 2006). El MP es altamente diverso y complejo y su incidencia sobre la salud depende de sus características físicas y químicas: concentración en masa, tamaño o diámetro aerodinámico, grado de penetración y retención respiratoria, composición química, solubilidad en fluidos biológicos, estado de salud de la población y meteorología de cada localidad impactada (Préndez, 1993). En la actualidad, en Chile son de interés regulatorio el MP sedimentable (MPS), el MP10 y el MP2,5 a partir de enero de 2012 (DS 12/2011.). Este último es de gran importancia en salud humana porque el MP de diámetro inferior a 1-2 $\mu \mathrm{m}$, e incluso menos, se deposita preferentemente en la región alveolar del pulmón, donde el paso al flujo sanguíneo de algunos elementos traza tóxicos bioacumulables, podría afectar la fisiología tanto del pulmón, como la del aparato circulatorio (OMS, 2006; Nel et al., 2006)

EI MP afecta la vegetación provocando la obturación de estomas y reducción de la fotosíntesis y del crecimiento (Dalmasso et al., 1997). No obstante, existe el rol de la vegetación como amortiguador sanitario en zonas pobladas (Manes et al., 2012). Dependiendo del origen, el depósito de aerosoles secundarios puede producir incrementos en la concentración de metales y alteraciones en el pH del suelo. El MP tiene una alta variabilidad espacial y temporal; sus concentraciones fluctúan con su ubicación espacial, estación del año, dirección del viento, siendo en general las concentraciones más elevadas en invierno que en verano, principalmente debido a las condiciones meteorológicas (Préndez et al., 2011).

El MP contiene diferentes elementos traza, algunos de ellos tóxicos para la población (Harrison y Yin, 2000). Diversos elementos traza al depositarse sobre el suelo, pueden ser lixiviados ó absorbidos por las plantas. Numerosas investigaciones indican que estos elementos pueden presentar un enriquecimiento anormal en la atmósfera, constituyendo un indicador para la identificación de sus fuentes (Fang et al., 2006; Préndez et al., 2007). Algunos elementos traza tienen norma primaria y/o norma secundaria, como se muestra en la tabla 1 para Chile y la comunidad europea, además de los límites recomendados por la OMS.

Otros elementos traza de interés en este estudio son cobre $(\mathrm{Cu})$, molibdeno $(\mathrm{Mo})$ y zinc $(\mathrm{Zn})$, esenciales para humanos, animales y plantas, y antimonio $(\mathrm{Sb})$ y talio (TI), elementos no esenciales. El Cu puede ser tóxico, pero no venenoso para el hombre. Está asociado a emisiones de las fundiciones de $\mathrm{Cu}$. La OMS no lo considera contaminante por exposición aérea por lo que no establece valores límite de referencia. El Mo no es tóxico para el hombre, los animales ni las plantas. Su presencia en el aire se asocia a fundiciones de cobre. La carencia de Mo, produce malformación en las hojas, necrosis y clorosis (SAG, 2005). La carencia de Zn puede causar problemas, pero su exceso también es perjudicial. Existe $\mathrm{Zn}$ liberado al ambiente por procesos naturales, pero la mayor parte es antropogénico. En los ambientes laborales se encuentra normado (ATSDR, 2005). El Sb y el TI son elementos de origen natural y antrópico. Son emitidos a la atmósfera por diversas industrias y se encuentran normados para ambientes laborales (ATSDR, 1995). 
Tabla 1: Normas de calidad para elementos traza en Chile, la comunidad europea (CE) y límites recomendados por la Organización Mundial de la Salud (OMS).

\begin{tabular}{|c|c|c|c|c|c|}
\hline \multicolumn{2}{|l|}{ Calidad del Aire } & $\mathrm{ng} / \mathrm{m}^{3}$ & \multicolumn{2}{|l|}{ Criterio } & \multirow{2}{*}{$\begin{array}{l}\text { Método } \\
\text { - Espectrometría de absorción atómica ISO } 9855 \\
\text { Aire ambiente Determinación del contenido } \\
\text { particulado de plomo en aerosoles captados en } \\
\text { filtros }\end{array}$} \\
\hline $\begin{array}{l}\text { DS } 136 / 2000 \text { Chile } \\
\text { Norma primaria } \\
\text { para plomo }(\mathrm{Pb})\end{array}$ & $\mathrm{Pb}$ & 500 & \multicolumn{2}{|l|}{ Media anual } & \\
\hline \multirow{8}{*}{$\begin{array}{l}\text { Norma primaria } \\
\text { OMS } \\
\text { (recomendación) } \\
\text { WHO, } 2000\end{array}$} & $\begin{array}{l}\text { As } \\
\text { (Según nivel } \\
\text { de riesgo) }\end{array}$ & $\begin{array}{l}66 \\
6,6 \\
0,6\end{array}$ & $\begin{array}{l}1: 10.000 \\
1: 100.000 \\
1: 1.000 .000\end{array}$ & $\begin{array}{l}\text { Media } \\
\text { anual }\end{array}$ & \multirow{8}{*}{ No especifica } \\
\hline & $\begin{array}{l}\mathrm{Ni} \\
\text { (Según nivel } \\
\text { de riesgo) }\end{array}$ & $\begin{array}{l}250 \\
25 \\
2,5 \\
\end{array}$ & $\begin{array}{l}1: 10.000 \\
1: 100.000 \\
1: 1.000 .000 \\
\end{array}$ & $\begin{array}{l}\text { Media } \\
\text { anual }\end{array}$ & \\
\hline & $\begin{array}{l}\mathrm{Cr}(\mathrm{VI}) \\
\text { (Según nivel } \\
\text { de riesgo) }\end{array}$ & $\begin{array}{l}2,5 \\
0,25 \\
0,025 \\
\end{array}$ & $\begin{array}{l}1: 10.000 \\
1: 100.000 \\
1: 1.000 .000 \\
\end{array}$ & $\begin{array}{l}\text { Media } \\
\text { anual }\end{array}$ & \\
\hline & $\mathrm{Cd}$ & 5 & \multirow{5}{*}{\multicolumn{2}{|c|}{ Media anual }} & \\
\hline & $\mathrm{Hg}$ & 1000 & & & \\
\hline & $\mathrm{Mn}$ & 150 & & & \\
\hline & $\mathrm{Pb}$ & 500 & & & \\
\hline & $\mathrm{V}$ & 1000 & & & \\
\hline $\begin{array}{l}\text { Directiva } \\
\text { 1999/30/CE }\end{array}$ & $\mathrm{Pb}$ & 500 & Media anual & & $\begin{array}{l}\text { - Espectrometría de absorción atómica ISO } 9855 \\
\text { Aire ambiente Concentración de Pb en aerosoles } \\
\text { captados sobre filtros } \\
\text { - Cualquier otro método con resultados } \\
\text { equivalentes al método anterior. }\end{array}$ \\
\hline \multirow{3}{*}{$\begin{array}{l}\text { Directiva } \\
\text { 2004/107/CE }\end{array}$} & As & 6 & \multirow{3}{*}{\multicolumn{2}{|c|}{ Media anual }} & \multirow{3}{*}{$\begin{array}{l}\text { - Muestreo MP } \mathrm{MP}_{10} \mathrm{EN} 12341 \\
\text { - Espectrometría de absorción atómica ó } \\
\text { espectrometría de masa ICP } \\
\text { - Los estados miembros podrán usar métodos } \\
\text { normalizados nacionales o ISO }\end{array}$} \\
\hline & $\mathrm{Cd}$ & 5 & & & \\
\hline & $\mathrm{Ni}$ & 20 & & & \\
\hline
\end{tabular}

\section{MATERIALES Y MÉTODO}

Área de Estudio

La zona de estudio se localiza en la sección baja e intermedia de la cuenca del río Aconcagua y abarca territorialmente las comunas de Concón, Quillota, La Cruz, La Calera, Nogales, Hijuelas, Limache, Llay Llay, Catemu y Panquehue. Todas ellas tienen estaciones de monitoreo permanente de la calidad del aire, salvo Nogales, donde se han realizado mediciones puntuales en el marco de la evaluación ambiental de proyectos. La tabla 2 muestra las variables meteorológicas y los contaminantes evaluados en las estaciones de monitoreo. Las estaciones de Concón Santa Margarita y Concón Sur cambiaron de ubicación, en los años 2000 y 2006, respectivamente. La topografía de la cuenca rodeada por el relieve de la cordillera de la costa, incide en el transporte de los contaminantes. Durante el día los flujos de aire SO transportan los contaminantes desde Concón, hacia las secciones más altas como Catemu y Panquehue; durante la noche el flujo de aire se invierte (CENMA, 2001). Las bajas temperaturas y velocidades promedio del viento y radiación solar en otoño e invierno facilitan la acumulación de contaminantes.

\section{Datos Usados}

De acuerdo a la tabla 2 se trabajó con alrededor de 2.400 .000 datos de calidad del aire y alrededor de 1.900 .000 datos de meteorología entregados por las empresas propietarias de las estaciones, basadas en la validación que efectúan las empresas administradoras de las estaciones. Se analizó la información disponible entre 1998 y 2008. Se evaluó el comportamiento de las variables señaladas para observar sus patrones diarios, mensuales y anuales. Para calidad del aire, el promedio diario se calculó con un mínimo de 18 promedios horarios válidos. Los promedios anuales se calcularon con un mínimo de $75 \%$ de promedios diarios válidamente medidos. Además, se efectuó una inspección visual del exterior de las estaciones monitoras y los aspectos técnicos de su emplazamiento se evaluaron aplicando la lista de chequeo de los requisitos normados para medición de partículas y gases (DS 61/2008). Las emisiones de las fuentes de la zona de estudio, se analizaron a partir de la declaración anual de emisiones de las empresas para el año 2006 (DS 138/2005). Además, se calculó la concentración promedio anual de los elementos traza presentes en el MP (informados a CONAMA, Región de Valparaíso), a objeto de observar tendencias. 
Tabla 2: Variables meteorológicas y contaminantes evaluados en las estaciones de monitoreo.

\begin{tabular}{|c|c|c|}
\hline Nombre estación & Calidad del aire (Contaminantes) & Meteorología \\
\hline Lo Campo & $\mathrm{MP} 10, \mathrm{SO}_{2}, \mathrm{As}, \mathrm{Cu}, \mathrm{Cd}, \mathrm{Hg}, \mathrm{Mo}, \mathrm{Mn}, \mathrm{Pb}, \mathrm{Zn}$ & \\
\hline Catemu & $\mathrm{MP} 10, \mathrm{SO}_{2}, \mathrm{As}, \mathrm{Cu}, \mathrm{Cd}, \mathrm{Hg}, \mathrm{Mo}, \mathrm{Mn}, \mathrm{Pb}, \mathrm{Zn}$ & \\
\hline Romeral & $\mathrm{SO}_{2}$ & \\
\hline $\begin{array}{l}\text { Santa Margarita } \\
\text { Chagres }\end{array}$ & $\mathrm{SO}_{2}$ & \\
\hline La Calera & $\begin{array}{l}\mathrm{MP} 10, \mathrm{SO}_{2}, \mathrm{O}_{3}, \mathrm{NO}_{2}, \mathrm{As}, \mathrm{Cd}, \mathrm{Hg}, \mathrm{V}, \mathrm{Tl}, \mathrm{Sb}, \mathrm{Ni}, \mathrm{Cr} \\
\mathrm{Pb}, \mathrm{Zn}\end{array}$ & Dirección y velocidad del viento \\
\hline Rural 1 & $\begin{array}{l}\mathrm{MP} 10, \mathrm{SO}_{2}, \mathrm{O}_{3}, \mathrm{NO}_{2}, \mathrm{As}, \mathrm{Cd}, \mathrm{Hg}, \mathrm{V}, \mathrm{Tl}, \mathrm{Sb}, \mathrm{Ni}, \mathrm{Cr} \\
\mathrm{Pb}, \mathrm{Zn}\end{array}$ & Dirección y velocidad del viento \\
\hline La Cruz & $\mathrm{MP} 10, \mathrm{SO}_{2}, \mathrm{O}_{3}$ & Dirección y velocidad del viento \\
\hline Hijuelas & $\begin{array}{l}\mathrm{MP} 10, \mathrm{SO}_{2}, \mathrm{O}_{3}, \mathrm{NO}_{2}, \mathrm{As}, \mathrm{Cd}, \mathrm{Hg}, \mathrm{Tl}, \mathrm{Sb}, \mathrm{Cr}, \mathrm{Pb}, \\
\mathrm{Zn}\end{array}$ & Dirección y velocidad del viento \\
\hline Rural 2 & $\mathrm{MP} 10, \mathrm{SO}_{2}$ & Dirección y velocidad del viento \\
\hline Poza & $\mathrm{MP} 10, \mathrm{SO}_{2}$ & \\
\hline $\begin{array}{l}\text { Concón Santa } \\
\text { Margarita }\end{array}$ & $\begin{array}{l}\mathrm{MP} 10, \mathrm{MP} 2,5, \mathrm{SO}_{2}, \mathrm{O}_{3}, \mathrm{NO}_{2}, \mathrm{HCT}, \mathrm{HCNM}, \mathrm{As} \\
\mathrm{Cu}, \mathrm{Cd}, \mathrm{V}, \mathrm{Ni}, \mathrm{Pb}\end{array}$ & $\begin{array}{l}\text { Dirección y velocidad del viento, } \\
\text { temperatura, Radiación solar, humedad } \\
\text { relativa, precipitaciones }\end{array}$ \\
\hline Concón Sur & $\mathrm{MP} 10, \mathrm{SO}_{2}, \mathrm{As}, \mathrm{Cu}, \mathrm{Cd}, \mathrm{V}, \mathrm{Ni}, \mathrm{Pb}$ & Dirección y velocidad del viento \\
\hline Colmo & $\mathrm{MP} 10, \mathrm{SO}_{2}, \mathrm{O}_{3}, \mathrm{NO}_{2}, \mathrm{HCT}, \mathrm{As}, \mathrm{Cu}, \mathrm{Cd}, \mathrm{V}, \mathrm{Ni}, \mathrm{Pb}$ & Dirección y velocidad del viento \\
\hline Las Gaviotas & $\mathrm{SO}_{2}$ & Dirección y velocidad del viento \\
\hline Bomberos & $\mathrm{MP} 10, \mathrm{SO}_{2}, \mathrm{O}_{3}, \mathrm{NO}_{2}, \mathrm{HCT}$ & Velocidad del viento \\
\hline San Pedro & $\mathrm{MP} 10, \mathrm{SO}_{2}, \mathrm{O}_{3}, \mathrm{NO}_{2}, \mathrm{HCT}$ & \\
\hline La Palma (Ex-Ucv) & $\mathrm{MP} 10, \mathrm{SO}_{2}, \mathrm{O}_{3}, \mathrm{NO}_{2}, \mathrm{HCT}$ & Dirección y velocidad del viento \\
\hline Quillota - Inp & $\mathrm{MP} 10, \mathrm{SO}_{2}, \mathrm{O}_{3}, \mathrm{NO}_{2}$ & Velocidad del viento, \\
\hline Cajón San Pedro & $\mathrm{MP} 10, \mathrm{SO}_{2}, \mathrm{O}_{3}, \mathrm{NO}_{2}$ & Velocidad del viento \\
\hline Limache & $\mathrm{MP} 10, \mathrm{SO}_{2}, \mathrm{O}_{3}, \mathrm{NO}_{2}$ & Velocidad del viento \\
\hline $\begin{array}{l}\text { Chagres } \\
\text { Meteorología }\end{array}$ & & Dirección y velocidad del viento \\
\hline $\begin{array}{l}\text { Melón } \\
\text { Meteorología }\end{array}$ & & $\begin{array}{l}\text { Dirección y velocidad del viento, tempera- } \\
\text { tura, Radiación. Solar, humedad relativa }\end{array}$ \\
\hline $\begin{array}{l}\text { Nehuenco } \\
\text { Meteorología }\end{array}$ & & $\begin{array}{l}\text { Dirección y velocidad del viento,, tempera- } \\
\text { tura, Radiación. Solar, humedad relativa }\end{array}$ \\
\hline
\end{tabular}

Para determinar el origen de los elementos traza medidos en cada estación monitora, se calcularon los factores de enriquecimiento, utilizando al menos un $67 \%$ de las concentraciones sobre el límite de detección. Se utilizó la fórmula aplicada en Préndez y Carrasco (1991) con las concentraciones de elementos traza en la corteza terrestre según Mason (1966) y las concentraciones en los suelos de la zona de estudio, obtenidos desde Gidhagen et al. (2000), Universidad Mayor (2005) y SERPRAM (2004). El impacto de las fuentes sobre cada estación de monitoreo se evaluó realizando correlaciones por pares y análisis de componentes principales (PCA), usando el software SPSS V17.0.

\section{Evaluación de Riesgos}

Se formuló y desarrolló una evaluación de riesgo cualitativo para establecer una zonificación en el área de estudio asociado a los contaminantes $\mathrm{MP}_{10}, \mathrm{SO}_{2}, \mathrm{NO}_{2}$ y $\mathrm{O}_{3}$ (Calderón, 2011). Se consideraron los tres componentes requeridos para que exista riesgo: existencia de un contaminante o fuente, presencia de receptores y vía de exposición. Se diseñó una matriz multi-criterio con el fin de sintetizar la información procesada, la cual consideró los siguientes factores: meteorología, calidad del aire y sus tendencias, magnitud de las emisiones, distancia de los receptores a fuentes más cercanas, población expuesta/vegetación expuesta y confiabilidad de los datos. Cada factor se jerarquizó de acuerdo a los criterios de valoración graduados en un escala de 1 a 4 , donde 4 tiene un mayor peso del factor en la composición del riesgo. El índice de riesgo para salud humana y para la vegetación se calculó a partir de las siguientes ecuaciones:

$$
\begin{aligned}
& I_{S P}=M E \cdot 1 F \cdot 2 F \cdot P E \cdot E C A \cdot M E T \cdot T C A \cdot C D \\
& I_{S P}=M E \cdot 1 F \cdot 2 F \cdot V E \cdot E C A \cdot M E T \cdot T C A \cdot C D
\end{aligned}
$$

donde,

$\mathrm{IR}_{\mathrm{SP}}$ : Índice de Riesgo para la salud de la población; $\mathbb{I R}_{\mathrm{V}}$ : Índice de Riesgo para la vegetación

ME: Magnitud de emisiones por comuna; 1F: Fuente más cercana; 2F: Segunda fuente más cercana; PE: Población Expuesta; VE: Vegetación Expuesta; ECA: Estado de la Calidad del Aire; MET: Meteorología; TCA: Tendencia de la Calidad del Aire; CD: Certidumbre de los Datos. 
Para ponderar la población expuesta se utilizó el Censo 2002 (www.ine.cl) y para la vegetación expuesta se interpretaron fotografías aéreas disponibles en www.sinia.cl y software Google Earth. Se obtuvo una caracterización del tipo de cultivo expuesto en cada comuna utilizando el Censo agropecuario y forestal 2007 (www.censoagropecuario.cl). Usando el software ArcGIS 9.1 del Sistema de Información Geográfica (SIG), se calculó el nivel de riesgo por contaminante para la población $\left(N R_{S P}\right)$ y para la vegetación $\left(N R_{V}\right)$ como el Log del índice de riesgo respectivo, esto es $\log \left(\mathrm{IR}_{\mathrm{SP}}\right)$ y $\log \left(\mathrm{IR}_{\mathrm{V}}\right)$. Se jerarquizó el riesgo según la tipología que se muestra en la tabla 3; el rango del riesgo es la diferencia entre los valores máximo y mínimo. Los resultados se representaron espacialmente mediante el SIG.

Tabla 3: Pauta para la jerarquización de los riesgos.

\begin{tabular}{|l|l|l|}
\hline Nivel de riesgo & Descripción & Definición \\
\hline Alto & $\begin{array}{l}\text { Tercio superior del rango de } \\
\text { Nivel de Riesgo }\left(\mathrm{NR}_{\mathrm{SP}} \mathrm{O} \mathrm{NR}_{\mathrm{V}}\right)\end{array}$ & $\begin{array}{l}\text { Existe mayor probabilidad de observar efectos adversos para la } \\
\text { salud de la población y/o la vegetación por la exposición al } \\
\text { contaminante. }\end{array}$ \\
\hline Medio & $\begin{array}{l}\text { Tercio medio del rango de Nivel } \\
\text { de Riesgo }\left(\mathrm{NR}_{\mathrm{SP}} \mathrm{O} \mathrm{NR}_{\mathrm{V}}\right)\end{array}$ & $\begin{array}{l}\text { Existe riesgo moderado para la salud de la población y/o la } \\
\text { vegetación por la exposición al contaminante. }\end{array}$ \\
\hline Bajo & $\begin{array}{l}\text { Tercio inferior del rango de Nivel } \\
\text { de Riesgo (NRSP O NRV })\end{array}$ & $\begin{array}{l}\text { Existe una menor probabilidad de observar efectos adversos para la } \\
\text { salud de la población y/o la vegetación por la exposición al } \\
\text { contaminante. }\end{array}$ \\
\hline
\end{tabular}

\section{RESULTADOS Y DISCUSIÓN}

\section{Variables Meteorológicas}

La información promedio muestra patrones diarios y estacionales para temperatura, intensidad del viento, radiación solar y humedad relativa. Se observa un patrón diario en la dirección del viento, el que se interna en la cuenca a partir del medio día, devolviéndose durante la madrugada con menor intensidad. Las precipitaciones se concentran en los meses de junio y julio. A lo largo de la cuenca se distinguen tres zonas meteorológicas. La figura 1 muestra algunos ejemplos de la dirección del viento en uno de las zonas sensibles de la cuenca, como es La Calera.

- Zona costera: desde Concón al sector de Colmo con un relieve bajo de altitud menor a $500 \mathrm{msnm}$; presenta temperatura más estable con una oscilación diaria promedio entre $12,8( \pm 2,7){ }^{\circ} \mathrm{C}$ y $18,4( \pm 3,2){ }^{\circ} \mathrm{C}$ en Concón (año 2005); la humedad relativa alta oscila entre $94,6( \pm 8,3) \%$ en la madrugada y $65,6( \pm 21,7) \%$ en la tarde (año 2005); tiene vientos moderados que no superan en promedio una intensidad máxima de 4,5 $( \pm 1,5) \mathrm{m} / \mathrm{s}$ (enero 2006 ) y predominan las componentes O, NON, ENE, E, ESE.

- Zona intermedia: desde el sector de San Pedro en Quillota hasta Hijuelas predominando cotas menores a 1000 msnm y encajonamiento hacia el sector de San Pedro y La Palma en Quillota y en La Calera; presenta temperaturas de mayor amplitud entre $10,3( \pm 3,5)$ y $20,2( \pm 4,8)^{\circ} \mathrm{C}$ (año 2005 ); la humedad relativa decrece al internarse en la cuenca y en La Calera oscila entre $76,9( \pm 9,1) \%$ y $52( \pm 15,5) \%$ (año 2005$)$; los vientos de menor intensidad en la mañana aumentan su intensidad hacia la tarde alcanzando un máximo promedio de $6,0( \pm 1,1) \mathrm{m} / \mathrm{s}$ (enero 2006), siguiendo la orientación de la cuenca en este sector.

- Zona cordillerana: desde Romeral en Hijuelas hasta Panquehue, con un relieve mayor y predominio de cotas inferiores a los $1500 \mathrm{msnm}$, con encajonamiento en el sector Santa Margarita con vientos de intensidad que alcanza los $10,0( \pm 1,2) \mathrm{m} / \mathrm{s}$ (enero 2006); predomina la dirección SSO del viento en Chagres.

\section{Contaminantes}

La información disponible para una década de $\mathrm{NO}_{2}, \mathrm{O}_{3}, \mathrm{SO}_{2}, \mathrm{MP10}$ y MP2,5, permite observar tendencias diferenciadas según el tipo de contaminante y la zona específica impactada, no así la presencia de un proceso general y extendido de deterioro de la calidad del aire de la cuenca. Las zonas con las mayores concentraciones de los contaminantes estudiados se localizan en las comunas con las mayores emisiones industriales de $\mathrm{NO}_{x}$, COV, $\mathrm{SO}_{x}$ y PTS, refinería de petróleo en Concón, termoeléctricas en Quillota, planta cementera de La Calera y fundición de cobre en Catemu, vinculándolas directamente a la calidad del aire y al impacto local. Sin perjuicio de lo anterior, para el caso de los promedios diarios de $\mathrm{NO}_{2}$ la alta correlación observada entre las diversas estaciones, supone una extensión de la zona de influencia de este contaminante que abarcaría desde Concón hasta La Calera. A su vez las altas concentraciones de $\mathrm{O}_{3}$ observadas en Limache entre 1999 y 2000, suponen una extensión hacia el este, en dirección a la sub-cuenca del estero de Limache. 


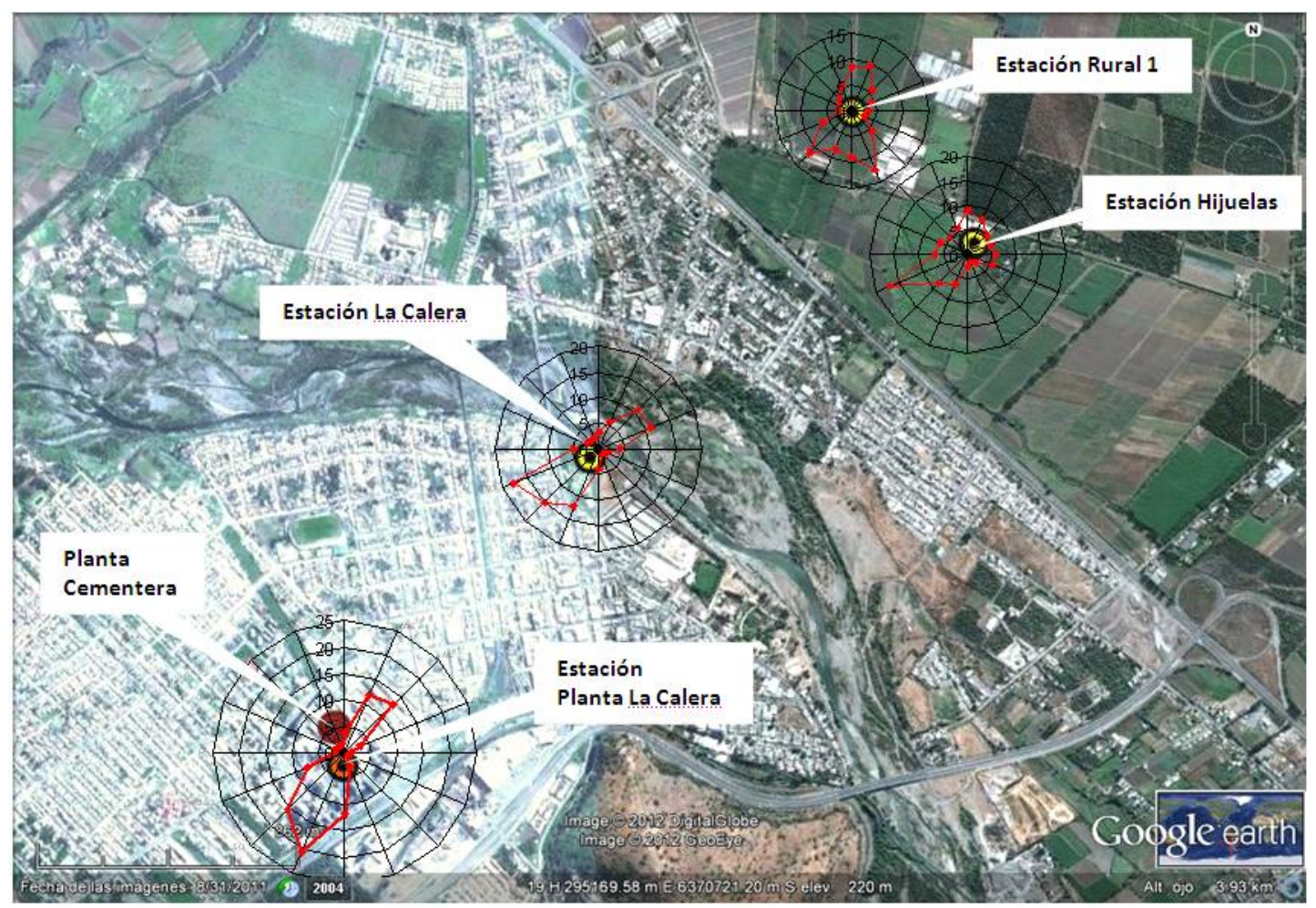

Fig. 1: Rosas de vientos para las estaciones de monitoreo del sector de la La Calera

La dispersión del MP10 depende de la velocidad de traslado de las masas de aire por la cuenca, dificultando su dispersión en las zonas aledañas a fuentes emisoras especialmente en invierno. La presencia de barreras físicas, preferentemente el relieve, obstaculiza mas el desplazamiento del MP aumentando su concentración. Es el caso de La Calera cuyas emisiones industriales, localizadas en un sector encajonado de la zona intermedia de la cuenca, explicarían el aumento sostenido de la concentración anual de MP10 a lo largo de todo el período estudiado, situándose sobre la norma primaria de calidad del aire. Condiciones de relieve similares se observan en Catemu donde las concentraciones de MP10 superaron la norma primaria anual, durante todo el período de estudio.

El cambio de combustible de gas natural a petróleo diesel en las centrales termoeléctricas entre 2004 y 2005, coincide con un incremento en la concentración de $\mathrm{NO}_{2}$ (1h) en las zonas vecinas de San Pedro y La Palma, especialmente evidente en 2007. Esto, sumado a las condiciones invernales desfavorables a la difusión y dispersión del gas, impacta los cultivos de la zona cuya capacidad de desintoxicación disminuye con las bajas temperaturas. En Concón, no se observan altas concentraciones de $\mathrm{NO}_{2}$, pero la alta humedad puede favorecer la formación y precipitación de aerosol de ácido nítrico el que puede afectar el pH del suelo local y hacia el interior de la cuenca. En 2010 la termoeléctrica San Isidro disminuyó su consumo de diesel al cambiar a gas natural licuado; en Nehuenco siguió predominando el uso del diesel (www.cne.cl).

La presencia de $\mathrm{NO}_{2}$ y $\mathrm{O}_{3}$ en la cuenca da cuenta de las emisiones de $\mathrm{NO}_{\mathrm{x}}$ y $\mathrm{COV}_{\mathrm{S}}$ antropogénicos y biogénicos en presencia de la radiación solar, la que además favorece la inestabilidad de las masas de aire incrementando la intensidad del viento durante la tarde y en verano; por el contrario, su ausencia determina tendencias inversas en el desplazamiento e intensidad de las masas de aire. Si bien no hay datos concretos de un aporte de $\mathrm{COV}_{\mathrm{S}}$ biogénicos es posible suponerlo desde las plantaciones de paltos y cítricos, reforzando una acumulación del $\mathrm{O}_{3}$, principalmente en verano. Esta situación no se observa en la zona urbana de La Calera la cual carece de zonas cultivables.

Es importante el rol de la velocidad y dirección del viento y del relieve en el transporte y acumulación del $\mathrm{O}_{3}$. Las altas concentraciones observadas en las estaciones de San Pedro, La Palma y Cajón San Pedro dan cuenta de ello. No obstante haberse registrado un incremento en las concentraciones promedio de $8 \mathrm{~h}$ para $\mathrm{O}_{3}$ desde la puesta en marcha del cambio energético en las centrales, situándose cercano a los límites normados $\left(120 \mu \mathrm{g} / \mathrm{m}^{3}\right.$, DS112/2001; $100 \mu \mathrm{g} / \mathrm{m}^{3}$, OMS 2005), se observa posteriormente en el trienio 2006-2008 un claro descenso del $\mathrm{O}_{3}$. Esta situación no se observa en La Calera donde las emisiones de $\mathrm{NO}_{x}$ desde los hornos cementeros son siempre elevadas. Con todo, no se observan excedencias a la normativa primaria para $\mathrm{NO}_{2}$ y $\mathrm{O}_{3}$, pero sí excedencia de los límites secundarios recomendados por la OMS (AOT40 para 5 días, aire húmedo: $400 \mu \mathrm{g} / \mathrm{m3}$ h, WHO, 2000). 
$\mathrm{El} \mathrm{SO} \mathrm{S}_{2}$ proviene de las fuentes industriales de la zona de estudio. La acumulación del $\mathrm{SO}_{2}$ depende de factores como la ventilación de la cuenca, la presencia de barreras geomorfológicas y la presencia de humedad. En general, la cuenca se presenta bien ventilada salvo algunos sectores, como es el caso del sector Santa Margarita localizado en una zona rural vecina a la fundición Chagres en Catemu. En dicha zona se observa, una acumulación de $\mathrm{SO}_{2}$, hacia la tarde, en verano, producto de su localización en las faldas de un sector montañoso flanqueado por dos cuencas bien ventiladas, la del Aconcagua y la de Catemu. Esto se podría explicar por la disminución de la humedad y por ende de la transformación $\mathrm{SO}_{2} \mathrm{a} \mathrm{H}_{2} \mathrm{SO}_{4}$ y/o algún sulfato secundario, tendiendo a acumularse a niveles de riesgo agudo para la población. No obstante, las concentraciones de $\mathrm{SO}_{2}$ han disminuido claramente en el tiempo, producto de las mejoras tecnológicas de abatimiento de emisiones; sin embargo, la presencia de $\mathrm{SO}_{2}$ y de As en el MP10 permiten suponer que aún existen emisiones no controladas que incidirían en la ocurrencia de algunos eventos horarios de concentraciones superiores al límite OMS de 500 $\mu \mathrm{g} / \mathrm{m}^{3}$. En Concón, la mayor acumulación de $\mathrm{SO}_{2}$ se produce en las mañanas cuando también disminuye la humedad relativa, lo que explicaría algunas excedencias episódicas al límite secundario de 1 hora.

La alta concentración de Cu observado en el MP10 de las estaciones de Concón, podría explicarse por el aporte de las emisiones de la refinería de Ventanas, considerando el sistema de vientos del área. Respecto de los elementos traza presentes en el MP10 de la cuenca del Aconcagua, el Mn es de origen natural, pero As, Cd, Cu, $\mathrm{Mo}, \mathrm{Pb}, \mathrm{Zn}$ y $\mathrm{Ni}$, son antropogénicos; las fuentes emisoras se sitúan en el área de estudio y corresponden a la Fundición Chagres, Planta Cemento Melón, Complejo Nehuenco-San Isidro y Empresa Nacional de Petróleo. No se descarta además en las zonas agrícolas la presencia de As de origen fitosanitario, así como de $\mathrm{Pb}$ desde fuentes móviles. El As en La Calera pudiera deberse a la influencia de alguna de las fundiciones de $\mathrm{Cu}$.

\section{Evaluación de Riesgo}

La figura 2 muestra los niveles de riesgo para la salud de la población y la vegetación, obtenidos a partir de la aplicación del método elaborado para este trabajo y que cruza variables meteorológicas, antropogénicas y de exposición humana y vegetacional. Se observa que las zonas que presentan riesgo alto para la salud de la población son: 1) por $\mathrm{NO}_{2}$ las localidades de San Pedro y La Palma y las zonas urbanas de Quillota y La Calera; 2) por $\mathrm{O}_{3}$, las localidades de San Pedro y Colmo y las zonas urbanas de Quillota y La Calera; 3) por $\mathrm{SO}_{2}$, la zona de Chagres, particularmente la zona rural de Santa Margarita, la zona urbana de Catemu, la localidad de San Pedro y las zonas urbanas de Quillota y de Concón; 4) por MP10 las zonas urbanas de Catemu y La Calera.

Las concentraciones de As, presentan valores superiores al límite europeo de $6 \mathrm{ng} / \mathrm{m}^{3}$, en todas las estaciones estudiadas, siendo la zona más impactada Lo Campo en Panquehue y la zona urbana de Catemu. A su vez se observaron niveles de $\mathrm{Cr}$ superiores al valor recomendado por la OMS de $2,5 \mathrm{ng} / \mathrm{m}^{3}$ en La Calera, Hijuelas y Rural 1. Las zonas que presentan riesgo alto para la vegetación por $\mathrm{NO}_{2}, \mathrm{O}_{3}$ y $\mathrm{SO}_{2}$ son las localidades de San Pedro, La Palma y Colmo. Así mismo presentan riesgo alto por $\mathrm{SO}_{2}$ las zonas rurales de Santa Margarita, Romeral, Lo Campo y la zona urbana de Catemu. En la zona predominan cultivos de paltos, cítricos y vides, los cuales podrían presentar efectos adversos.

\section{CONCLUSIONES}

Los resultados señalan claramente la necesidad de declarar zonas saturadas y aplicar un plan de descontaminación a La Calera y Catemu, por superación de la norma primaria anual de MP10 (DS 59/1998), además de observarse riesgos altos a la población y/o la vegetación por otros contaminantes. La reciente publicación de la norma primaria para MP2,5 hace más urgente este requerimiento. La situación detectada en los alrededores de la fundición de Chagres debiera inducir a la autoridad a exigir un mayor control de las emisiones fugitivas de $\mathrm{SO}_{2}, \mathrm{MP} 10$ y elementos traza principalmente As, elemento que presenta niveles elevados en Lo Campo y Catemu.

Existe una brecha importante entre los límites de las normas primarias de calidad del aire chilenas y los límites recomendados por la OMS. Faltan normas secundarias para aire para $\mathrm{NO}_{2}, \mathrm{O}_{3}$ y MP. Tampoco hay normas primarias para elementos traza, excepto $\mathrm{Pb}$ (DS 136/2000). Los resultados obtenidos revelan la necesidad de contar con normas de calidad para As, Cr, Ni, Cd, Zn y Mo. La importancia de la zona como productora de frutos de exportación debiera motivar a la autoridad a exigir un mayor control de las emisiones de las fuentes en el área de estudio, ya sea a través de cambios de combustibles o abatimiento de emisiones. Avances en robustecer el Sistema Nacional de Calidad del Aire como base de datos de acceso público y en tiempo real de las mediciones, permitirían implementar mejoras en la gestión atmosférica de otras cuencas del país. El método elaborado es una aproximación para la gestión ambiental a escala de cuenca y podría robustecerse incorporando nuevos factores, ajustando los considerados y cotejándolos con otros actores relevantes. 


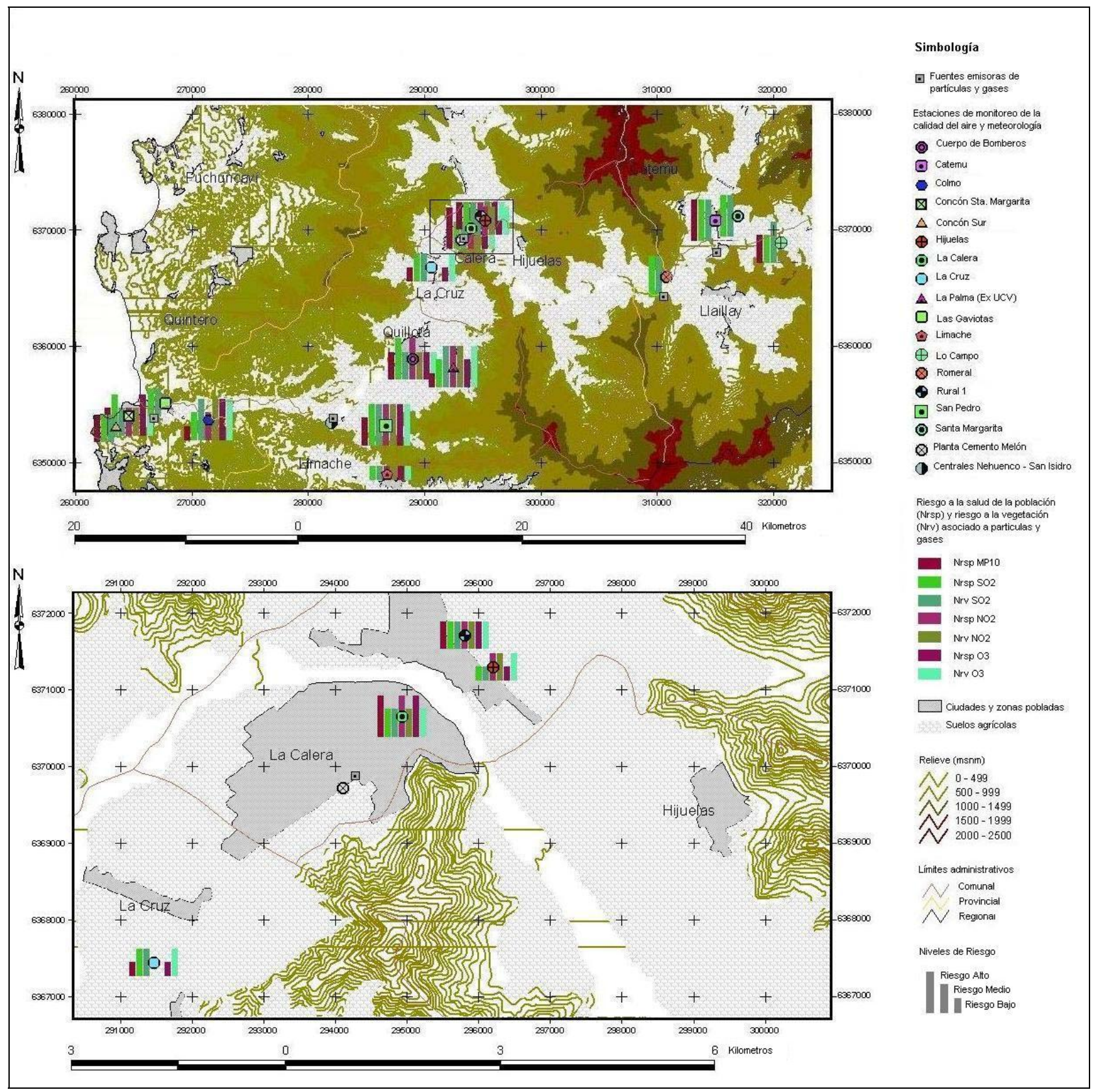

Fig. 2: Niveles de riesgo para la salud de la población y la vegetación

\section{AGRADECIMIENTOS}

Al Centro Nacional del Medio Ambiente por el financiamiento para la publicación del artículo

\section{REFERENCIAS}

ASIVA (Asociación de Industriales de Valparaíso y Aconcagua), Caracterización Económica de la Provincia de Quillota. Departamento de Estudios de la Asociación de Empresas, V región. (2011).

ATSDR (Agency for Toxic Substances and Disease Registry), División de Toxicología. Ficha informativa sobre Antimonio (1995).

ATSDR (Agency for Toxic Substances and Disease Registry), División de Toxicología. Ficha informativa sobre Cinc. (2005).

Cakmak, S., Dales, RS and Blanco, C. Air pollution and Mortality in Chile: Susceptibility among the Elderly. Environmental Health Perspectives. 115(4): 524-527 (2007). 
Calderón, Víctor. Evaluación de riesgos a la salud humana y vegetacional debido a la presencia atmosférica de contaminantes $\mathrm{MP}_{10}, \mathrm{MP}_{2,5}, \mathrm{SO}_{2}, \mathrm{NO}_{2}, \mathrm{O}_{3}$ y elementos traza en la cuenca del Aconcagua, Chile. Tesis de Magister en Gestión y Planificación Ambiental, U. de Chile (2011).

Calfapietra, C., Fares, S., Loreto, F., Volatile organic compounds from Italian vegetation and their interaction with ozone. Environmental Pollution 157: 1478-1486 (2009)

CENMA.(Centro Nacional del Medio Ambiente), Diagnóstico Integral de la Contaminación Atmosférica en la Macrozona Central de Chile. Santiago. CENMA y CONAMA, 148 p. (2001).

Dalmasso, A., Candia, R., Llera, J., La vegetación como indicadora de la contaminación por polvo atmosférico. Multequina - Instituto argentino de investigación de las zonas áridas, 6: 91-97 (1997).

DS 59 Decreto Supremo № 59. Norma de calidad primaria para material particulado respirable MP10, en especial de los valores que definen situaciones de emergencia. Ministerio Secretaría General de la Presidencia de la República, Santiago, Chile (1998).

DS 136 Decreto Supremo № 136. Norma de calidad primaria para plomo en el aire, Santiago, Chile (2000).

DS 138 Decreto Supremo № 138. Declaración de emisiones que indica, Santiago, Chile ( 2005).

DS 61 Decreto Supremo № 61. Reglamento de Estaciones de Medición de Contaminantes Atmosféricos, Santiago, Chile (2008).

DS 12 Decreto Supremo No 12. Norma Primaria de Calidad Ambiental para Material Particulado Fino Respirable MP2.5, Santiago, Chile (2011).

Fang, G., Wu, Y., Chang, S., Huang, S. y Rau, J. Size distributions of ambient air particles and enrichment factor analyses of Metallica elements at Taichung Harbor near the Taiwan Strait Atmospheric Research. 81: 320-333 (2006).

Fernandez, F., Ternero, M., Fernandez. A.J., Gutierrez, A., Physical speciation of arsenic, Mercury, lead, cadmiun and nickel in inhalable atmospheric particles. Analytica Chimica Acta. 524: 33-40 (2004).

Gallardo, L., Trazas atmosféricas y su modelación. En: Morales, R. (Ed). Contaminación atmosférica urbana: episodios críticos de contaminación ambiental en la ciudad de Santiago. Editorial Universitaria p. 83 - 104 (2006).

Gidhagen, L., Oyola P., Schmidt-Thomé, P., Kahelin, H. y Langner, J., Determinación de línea base nacional de contenido de arsénico en material particulado respirable. CONAMA., (2000). http://www.bvsde.opsoms.org/sde/ops-sde/bvsde.shtml

Gramsch, E., Cereceda-Balic, F., Oyola, P. y Von Baer, D., Examination of pollution trends in Santiago de Chile with cluster analysis of PM10 and Ozone data. Atmospheric environment. 40: 5464-5475 (2006).

Harrison, R. y J. YIN, Particulate matter in the atmosphere: which particle properties are important for its effects on Health?. The Science of the Total Environment. 249: 85-101 (2000).

Manes, F., Incerti, G., Salvatori, E., Costanza, R., Vitale, M., and Ricotta, C. Urban ecosystem services: tree diversity and stability of tropospheric ozone removal. Ecological Applications, 22(1): 349-360 (2012).

Mason, B., Principles of geochemistry. 3rd Edition, J. Wiley and Sons, NY. (1966).

Nel A, Xia T., Mädler, L., Li, N. Toxic potential of materials at the nanolevel. Science 311: 622-627 (2006).

OMS (Organización Mundial de la Salud) Guías de Calidad de Aire de la OMS relativas al material particulado, el ozono, el dióxido de nitrógeno y el dióxido de azufre, actualización 2005. Ginebra, Suiza, Organización Mundial de la Salud. (2006).

Préndez, M. Características de los contaminantes atmosféricos Capítulo 5. In: Contaminación Atmosférica de Santiago: Estado Actual y Soluciones. Sandoval, Préndez y Ulriksen (Eds). Editorial Cabo de Hornos. (1993). 
Préndez, M. y Carrasco, A., Element distribution of some soils of continental Chile and the Antarctic peninsula. Projection to atmosferic pollution. Water, Air and Soil Pollution, 57-58: 713-722 (1991).

Préndez, M., Corvalán, R. y Cisternas, M. Caracterización física y química del material particulado de fuentes estacionarias: incidencia sobre la aplicación de un sistema de compensación de emisiones en la Región Metropolitana de Santiago. Int. Tecnol. Vol 18 (2), 93-103 (2007).

Préndez, M., G. Alvarado and I. Serey. Chapter 15: Some Guidelines to Improve Air Quality Management in Santiago, Chile: from Commune to Basin level. In: Air Quality Monitoring, Assessment and Management. p. 305-328. N. Mazzeo Editor INTECH Open Access Publisher, 378 pages (2011).

Rana, S.V.S. Environmental Pollution, Health and Toxicology. Department of Zoology Ch. Charan Singh University Merrut, India, 250 p Editor.Alpha Science International Ltd. (2007).

SAG (Servicio Agrícola y Ganadero) Criterios de calidad de suelo agrícola, Chile. Servicio Agrícola y Ganadero. (2005).

http://bibliotecadigital.sag.gob.cl/documentos/medio_ambiente/criterios_calidad_suelos_aguas_agricolas/pdf _suelos/1_portada_indice.pdf

Seguel,R.J., Morales, R.G.and Leiva, M.A. Estimations of primary and secondary organic carbon formation in PM2.5 aerosols of Santiago City, Chile. Atmospheric Environment 43, 2125-2131 (2009).

SERPRAM (Servicios y Proyectos Ambientales).Campaña de monitoreo de línea de base de suelos y tejidos vegetales. Cemento Melón S.A. y SERPRAM (2004).

Tak Sun Yu, I., Y. hui Zhang, W. Wai San Tam, Q. Hua Yan, X. jun Xun, W., Wen Jun Ma, L. Wei Tian, L. Ah Tse, Y. jun Xu, X. Qian Lao. Effect of ambient air pollution on daily mortality rates in Guangzhou, China. Atmospheric Environment 46, 528-535 (2012).

Universidad Mayor. Desarrollo de una metodología para la evaluación y mitigación de la contaminación de aguas y suelo: Aplicación a la cuenca del río Aconcagua. Santiago, SAG y Escuela de Medicina Veterinaria, Facultad de Ciencias Silvoagropecuarias, (2005).

WHO, Air Quality Guidelines for Europe. Copenhagen, Regional Office for Europe, WHO regional Publications, European Series, No 91, (2000). 
